\title{
Basophils and IgE: Linking the Allergic Environment to Autoimmunity
}

\author{
Nicolas Charles ${ }^{\dagger}$ and Juan Rivera* \\ Laboratory of Molecular Immunogenetics, National Institute of Arthritis and Musculoskeletal and Skin Diseases, Na- \\ tional Institutes of Health, Bethesda, Maryland 20892, USA
}

\begin{abstract}
As outlined in some of the accompanying articles in this issue, the role of the basophil as an effector cell in allergy and in host defense (particularly to parasites) has long been recognized. However, recent advances advocate for the basophil as an immunomodulatory cell that can promote naïve $\mathrm{CD} 4^{+} \mathrm{T}$ cell commitment to $\mathrm{Th} 2$ cell differentiation. While this is in keeping with the concept that the basophil is important in an allergic environment, new discoveries suggest that basophils may be immunomodulatory beyond the context of allergic disease. Here we mainly discuss our own work, which provides a new paradigm for the role of basophils beyond allergy. Our findings demonstrate the importance of autoreactive IgE's, IL-4 and basophils in promoting autoantibody production and the development of lupus nephritis. The conclusions drawn are based on studies in a mouse model ( $\mathrm{Cyn}^{-/}$mice) of spontaneous systemic lupus erythematosus (SLE)-like disease as well as from analysis of the relationship between disease activity in SLE patients and their levels of autoreactive IgE's and activated basophils with antigen presenting capability. The findings demonstrate a link between the Th2 environment and autoimmunity and provide new areas of investigation with therapeutic potential.
\end{abstract}

Keywords: Allergy, Basophils, IgE, Lupus, Lyn.

\section{BASOPHILS: ARE THEY A LINK BETWEEN THE ALLERGIC ENVIRONMENT AND AUTOIMMU- NITY?}

Associated cellular and molecular mechanisms described in parasite infections and allergic disorders have demonstrated the fundamental importance of Th2 cell differentiation and function in these diseases $[1,2]$. Indeed, Th2 cytokines (like IL-4, IL-5, IL-13, IL-6, IL-10, TSLP, etc.) and Th2 immunoglobulins are associated in mouse (IgA, IgG1 and $\operatorname{IgE}$ ) and humans (IgA, IgG4 and IgE) with some parasitic infections and allergies [2]. In both mouse and human, the basophil has been linked to allergic disease [3, 4], with the demonstration in mouse models of a key role for these cells in chronic allergic inflammation [4] and in IgGmediated systemic anaphylaxis [5]. The recognition that mice, like humans, have circulating basophils has ushered in a new era for this long ignored cell type. Mouse models have allowed the exploration of basophil function in vivo as well as the investigation of the molecular factors important in basophil differentiation and function. As outlined in more detail in other chapters of this issue, recent advances (reviewed in $[2,6,7]$ ) have made it clear that basophils can cause Th2 cell differentiation in vivo thus demonstrating that these cells have an immunomodulatory role in addition to the long recognized effector role in allergic disease.

For a number of years, we investigated the molecular mechanisms that underlie the function of the high affinity $\operatorname{IgE}$ receptor ( $\mathrm{Fc \varepsilon RI}$ ), which is expressed on mast cells and basophils [8]. This effort has led us to explore the role of

*Address correspondence to this author at the NIAMS-NIH, Building 10, Room 13C103, Bethesda, MD. 20892-1930, USA; Tel: 301-496-7592; Fax: 301-480-1580; E-mail: juan_rivera@nih.gov

'Present address: Institut National de la Santé et de la Recherdie Médicale INSERM-699 Faculté de Médecine Xavier Bichat-Université Paris 7 Denis Diderot, 16 rue Henri Huchard, 75018, Paris, France; Tel: +33157277306; E-mail: nicolas.charles@inserm.fr
Lyn kinase, the kinase that phosphorylates FceRI and initiates signal transduction, in mast cell and basophils [8,9]. Interestingly, our efforts [10-12], as well as those of others [13-17] led to the recognition that a deficiency in Lyn kinase does not necessarily abrogate allergic responses and, in fact, these mice are hypersensitive to an allergic challenge [10, 18-20]. Lyn kinase is a member of the Src family of protein tyrosine kinases (SFK) and while expressed in most hematopoietic cells it is not expressed in T cells [21]. Nonetheless, the hypersensitivity of Lyn-deficient mice to allergic stimuli provided a strong indication that these mice showed an immune system imbalance whereby $\mathrm{Th} 2$ responses were highly favored. Studies characterizing the immune cell characteristics and distribution in Lyn-deficient mice demonstrated that these mice had a constitutive induction of naïve $T$ cell differentiation to Th2 cells [22]. This was dependent on the presence of IL-4 and IgE in these mice but was shown not to be dependent on the presence of mast cells. This led us to explore basophils and whether they might play a role in the observed Th2 skewing. Lyn deficient mice were found to develop a marked peripheral basophilia, with as much as a four fold increase in circulating basophils in unchallenged mice. Importantly, we discovered that an in vivo depletion of the basophils led to a complete stop in the differentiation of naïve $T$ cells to Th2 cells [22]. Collectively, our findings argued that basophils and IgE were key components in the Th2 skewing of Lyn-deficient mice and this axis was essential to the exacerbated allergic phenotype seen in these mice.

It is also well known that in late life Lyn-deficient mice develop a disease that resembles human systemic lupus erythematosus (SLE) $[18,19]$. The features of this disease include increased plasma cell numbers, the production of autoantibodies, the presence of vast immune complex depostion in the kidney, and the development of an inflammatory lupus nephritis-like disease that ultimately leads to renal failure. This late life phenotype (usually $>20$ weeks of age) 
is preceded by the allergic-like (Th2) phenotype (which occurs within 4-6 weeks of age). Thus, this presented an opportunity to explore whether the Th2 phenotype can contribute to the development of a lupus-like disease. While Th2 responses have been described in SLE patients [23], the presence and the role of these responses is unexplored and the concept of Th2 contribution to SLE is controversial, given that Th1, Th17, and loss of regulatory $\mathrm{T}$ cell function have been involved [24-30].

In this brief review, we describe new findings that argue for a Th2 component in the development of lupus nephritis. We detail our findings in the context of what is known for the role of Th2 responses in human SLE. Most importantly, and consistent with previous studies [31-35], the findings suggest that basophils are key regulators of humoral immune responses and that dysregulation of their function can have consequences that go well beyond the development or exacerbation of allergic disease.

\section{THE IMMUNOMODULATORY ROLE OF BASO- PHILS IN TH2 CELL DIFFERENTIATION AND AL- LERGIC RESPONSES.}

While this topic will be covered in depth in the accompanying articles, here we briefly outline the major points in basophil regulation of Th2 cell differentiation in order to discuss our findings in the context of the previous work [3135].

Th2 type cytokines, like IL-4, are known to induce the differentiation of naïve $\mathrm{CD} 4^{+} \mathrm{T}$ cells into $\mathrm{Th} 2$ cells that also produce IL-4, through the induction of transcription factors such as STAT6 and GATA-3 [36]. Th2 cells play an important role in host defense to some extracellular pathogens (parasites or bacteria) and in humoral immune response, but at times they can also have deleterious effects such as in the case of allergies or asthma [36]. Th2 cell responses dampen the ability to induce Th1 cell differentiation and in some cases, like in autoimmunity, this may be beneficial [37]. While it is clear that naïve $\mathrm{CD} 4^{+} \mathrm{T}$ cells can differentiate into Th2 cells [38], the cellular origin of the IL-4 inducing this commitment is still controversial. The IL-4 derived from the Th2 cell is able to amplify Th2 differentiation [38], but the initial IL-4 signals must come from another cell type that is able to distinguish a Th2-inducing challenge from others. Dendritic cells (DCs) are innate immune cells that have been shown to induce naïve $\mathrm{CD}^{+} \mathrm{T}$ cell to commit to Th2 differenation, in an IL-4 independent manner, both in vitro and in vivo [39]. However, it is also well known that DCs are not very potent IL-4 producers [39]. Thus it appears that their immunomodulatory function may function in collaboration with other cells that are also able to produce IL-4 and thus induce $\mathrm{Th} 2$ cell differentiation.

In humans and mice, basophils are known to produce copious amounts of Th2 cytokines, particularly IL-4, IL-6, IL13, and TSLP [3]. Basophils can produce high amounts of IL-4 to various stimuli, including FceRI stimulation, IL-3, and IL-33 treatment $[3,40]$. This has led to the thinking that basophils might play a role in the initiation or amplification of Th2 cell differentiation. However, the absence of appropriate tools and information on basophil biology and function, and the slow recognition that mice also have circulating basophils, has hampered their study in vivo. Recent reports $[34,41]$ have described new tools facilitating the study of basophil involvement in health and disease. Identification of cell surface markers and the development of methods to deplete the basophil in vivo through use of specific antibodies allowed a considerable advance. Antibody-mediated basophil depletion can be accomplished by in vivo injection of antibodies recognizing either CD200R3 (clone Ba103) or the high affinity IgE receptor alpha chain (FceRI $\alpha$, clone MAR-1) [34, 41]. The basophil depletion is thought to occur via cytolysis through complement activation. However, the precise mechanism still needs to be uncovered. Moreover, what secondary effects might occur after these injections are unknown. Nevertheless, the antibody-mediated depletion of basophils does not deplete mast cells (which also express the Fc\&RI $\alpha$ ) and does not induce detectable changes in the serum levels of cytokines [34, 41]. Such methods are highly useful to analyze the role of basophils in short term immunologic reactions.

These methods have proved quite useful in studying the immunomodulatory role of basophils in allergic reactions $[34,41]$. Papain immunization of mice is known to induce Th2 cell differentiation and promote papain-specific IgE and IgG1 production, hallmarks of a Th2 response [34]. Using the approach of antibody-mediated depletion of basophils, Sokol et al. demonstrated that basophils were key players in Th2 cell differentiation induced by subcutaneous injection of papain. Both IL-4 and TSLP produced by basophils were found to be key factors in promoting Th2 cell differentiation [34]. IL-4, IL-6 and IL-13 are also involved in a wide variety of immune regulation, including B cell immunoglobulin (Ig) switch recombination. These cytokines are known to facilitate $B$ cell switch to $\operatorname{IgA}, \operatorname{IgG}$ and $\operatorname{IgE}$ [42-44]. Moreover, IL-4 and IL- 6 are known to induce B cell maturation towards memory B cells and plasma cells [43, 44]. Denzel et al. recently showed that basophils were significant contributors in memory humoral immune responses by their ability to secrete IL-4 and IL-6 [35]. After sensitization, basophils were able to induce $\mathrm{B}$ cell maturation and lead to an efficient memory humoral response upon a second encounter with the antigen. Thus, this study demonstrated another key immunomodulatory role for basophils as contributors to humoral memory responses [35].

Additional work from the laboratory of H. Karasuyama has also shown an immunomodulatory role for basophils in promoting IgE-dependent chronic allergic inflammation (IgE-CAI) in a mouse model $[4,41]$. Basophils were shown to be responsible for promoting the chronic phase of allergic inflammation, including the recruitment of other immune cells type $[4,41]$. However, in more acute allergic responses, the effector function of basophils seems dominant as demonstrated by their key role in IgG-mediated systemic anaphylaxis through the release of large amounts of platelet activating factor (PAF) [5]. Thus, it seems that the basophils effector or immunomodulatory roles may be dominant depending on the immune challenge. One factor that may be key in distinguishing the effector from the immunomodulatory phase of basophils may be their ability to present antigen. Three recent studies demonstrated that basophils can process antigen and present it via upregulation of class II major histocompatibility complex [31-33]. In the peripheral blood, basophils are not known to express MHC-II, making this a 
sophils are not known to express MHC-II, making this a feature for their recognition in human peripheral blood [45]. Thus, in the periphery where they can play a role in acute IgG-mediated anaphylaxis, expression of MHC-II is not apparent. Yoshimoto et al., Sokol et al. and Perrigoue et al., showed that human and mouse basophils upregulate MHC-II expression in the secondary lymphoid tissues where they can serve as professional antigen presenting cells in the context of Th2 cell responses. These features make the basophil an important player in the immune system as both an effector and a regulatory cell that can initiate $\mathrm{Th} 2$ cell differentation [31-33] and amplify humoral responses [35].

\section{THE BASOPHILS BEYOND ALLERGY}

Like mast cells, the basophils role in the immune system has been mainly focused on its role in acute models of allergic reactions or on chronic models of parasitic infection where they appear to be important upon a second infection with the parasite [46]. However, if the basophil functions as an immunomodulatory cell, one might predict that there would be instances where the basophil might play an important role in immune diseases other than allergic disease. We and others $[22,47]$ recently reported that genetically modified mice, namely $I R F 2^{-/}$and $L y n^{-/}$mice, develop a peripheral basophilia and have a constitutive Th2 skewing of the $\mathrm{CD}^{+} \mathrm{T}$ cells. Both genes appear to play a negative regulatory role in peripheral basophil proliferation. The mechanism(s) leading to the constitutive Th2 skewing in vivo was associated with basophils, based on their numbers or on their increased sensitivity to different in vivo stimuli (like IL-3 and IgE-dependent stimulation). As $I R F 2^{-/-}$and $\mathrm{Lyn}^{-/-}$mice showed the development of skin inflammation [48] or of an SLE-like autoimmune disease [18, 19, 49], respectively, these models offer the unique opportunity to explore the role of basophils beyond allergic disease.

\section{BASOPHILS AND AUTOIMMUNE DISEASES}

The involvement of basophils in autoimmune disease has been documented in autoimmune urticaria where the development of autoantibodies to FceRI $\alpha$ have been described and shown to induce basophil activation [50-52]. In atopic dermatitis and other allergic-related skin diseases, basophils and mast cells are known to be involved in promoting the allergic environment leading to allergic cutaneous disease. Nonetheless, there is growing awareness that mast cells can contribute to autoimmune arthritis in some mouse models [53]. In this particular disease, as well as in most of the autoimmune diseases, the effector and immunomodulatory function of the mast cell leads to the initiation of inflammation. The enhancement of inflammation in autoimmune disease, which is the most deleterious component causing tissue damage upon its persistence, can be attributed to Th1 and Th17 cell responses. These $\mathrm{CD}^{+} \mathrm{T}$ cell subsets are believed to be the main effector cells involved in chronic inflammation during autoimmune diseases [54]. As always with such blanket categorization, one is tempted to think that other cell types are not involved in these diseases. However, as mentioned for urticaria and atopic dermatitis, IgE and its high affinity receptor FceRI, which is expressed on mast cell and basophils, are known to be involved in these diseases. In these aforementioned diseases, a Th2 component is well described and the basophil through its immunomodulatory and effector responses contributes to the development of the autoimmune symptoms. While IgE is clearly recognized as a "Th2 marker" in human pathologies there have been multiple reports $[23,55,56]$ where autoimmune patients are described to have increased levels of $\mathrm{IgE}$ but are studied in the context of their Th1 and Th17 responses [57]. While the importance of Th1- and Th17-driven responses in the development of autoimmunity is evident, it also seems unlikely that the presence of a Th2 response would not be contributory to the disease. Moreover, in highly complex diseases such as SLE (which might be characterized as a collection of different immunological disorders) it seems likely that the disease results from any imbalance of immune homeostasis [24-30].

From an epidemiological perspective, a parallel between allergy and autoimmunity is evident. Indeed, the incidence of both types of diseases are similarly increasing in developed countries [58]. As previously mentioned, allergy and autoimmunity share a number of common features. In both cases there can be manifestations that are associated with the production of autoantibodies and circulating immune complexes (CIC) formation [59]. Some studies have revealed a common structure of some allergens and autoantigens [60], with a developing hypothesis that many environmental allergens have common structural conformations with self-antigens [60]. This hypothesis could explain the development of certain autoimmune disease such as the chronic urticaria. However, in other autoimmune diseases (such as SLE) where increased IgE levels have been described, these were not associated with atopic features or increased allergic disease in this population [23]. Thus, high levels of circulating IgE may simply reflect the presence of a Th2 response in a nonallergic setting. Given the recent advances outlined above on the role of basophils in Th2 cell differentiation, this could suggest the contribution of basophils in autoimmune pathologies.

Recently it was reported that IgD-mediated basophil activation is relevant to some immune disorders such as HIDS (hyper IgD syndrome), TRAPS (TNF-receptor associated periodic syndrome) or MWS (Muckle-Wells syndrome), all of them being chronic autoinflammatory diseases [61]. The findings suggested that IgD-mediated basophil activation (through a receptor yet to be identified), both in humans and mice, was involved in B cell homeostasis and antibody production, particularly IgA and IgG [61]. Thus, in this context, the basophil has the potential to be involved in autoimmune disorders, where autoantibodies and abnormal levels of $\operatorname{IgA}$, $\mathrm{IgG}$ and $\mathrm{IgE}$ are found. Indeed, beyond the B cell abnormality and a subsequent humoral dysregulation seen in autoimmune disorders like SLE, the presence of Th2 type Ig's like IgEs strongly argues for the potential role of an IL-4 secreting cell (Th2 type cell) that influences the humoral response in this pathological setting.

\section{THE LYN DEFICIENT MOUSE MODEL: FROM AL- LERGY TO AUTOIMMUNITY}

As previously mentioned, Lyn kinase is expressed in most of the hematopoietic cells but not in T cells [21]. The activity of this kinase Lyn is required downstream of various 
receptors including cytokine receptors (such as IL-3 and IL4), tyrosine kinase receptors (such as CD117 or c-Kit), B cell antigen receptor and $\mathrm{Fc}$ receptors [62]. As the kinase responsible for phosphorylation of FceRI, one might have predicted that ablation of its expression or activity would prevent mast cell activation. However, we and others demonstrated that Lyn kinase has dual function as positive and negative regulator of FceRI signaling $[10,62]$. Thus, in the context of increased expression of Fyn kinase (such as seen in mast cells from $129 \mathrm{~Sv}$ or Balb/c mice), the absence of Lyn causes increased mast cell responses and exacerbation of allergic disease [12, 63]. Interestingly, Lyn kinase also seems to control mast cell proliferation or survival in vivo, since $\mathrm{Lyn}^{-/}$mice have an increased number of peritoneal mast cell, which is IgE-dependent and is seen early in life [10, 64]. Within 5-6 weeks of age $\mathrm{Lyn}^{-/-}$mice showed increased responsiveness to bronchial challenges (in an asthma model) and to passive systemic anaphylaxis $[10,20] . \mathrm{Lyn}^{-/-}$mice also have elevated levels of $\operatorname{IgM}, \operatorname{Ig} \mathrm{A}$ and $\operatorname{IgE}$ in their serum, and this phenotype also develops in early life and is associated with a profoundly altered peripheral B cell compartment and increased numbers of plasma cells $[18,19]$. The B cells from these mice were described to be hypersensitive to IL-4 and CD40L stimulation [65], suggesting a mechanism for the strong Th2 responses seen in these mice.

\section{The Th2 Skewing of $\mathrm{Lyn}^{-/-}$Mice is IL-4-, IgE-, and Baso- phil-Dependent}

To directly assess if $\mathrm{Lyn}^{-/-}$mice were Th2 skewed we analyzed $\mathrm{CD}^{+}$splenocytes (in the absence of any stimulation) from unchallenged mice and found a large number of IL-4 producing $\mathrm{CD}^{+}{ }^{+} \mathrm{T}$ cells, confirming a strong constitutive Th2 skewing [22]. Given the allergic bias of these mice, we set out to explore the role of IL-4, IgE and mast cells in the constitutive Th2 bias by generating mice deficient for both IL-4 and Lyn (Il-4 $\left.4^{-/} \mathrm{Lyn}^{-/-}\right), \operatorname{IgE}$ and Lyn $\left(\operatorname{Igh} 7^{-/} \mathrm{Lyn}^{-/-}\right)$, and mast cells and Lyn $\left(W^{5 h} / W^{S h} L y n^{-/-}\right)$. Strikingly, mice that were deficient in Lyn and IL-4 $\left(\mathrm{Il}-4^{-/-} \mathrm{Lyn}^{-/}\right)$or $\operatorname{IgE}\left(\mathrm{Igh} 7^{-/-}\right.$ $\mathrm{Lyn}^{-/}$) showed no Th2 skewing. In contrast, mice that were deficient in Lyn and mast cells $\left(W^{S h} / W^{S h} L y n^{-/-}\right)$had a similar constitutive Th2 skewing as in $L_{y n}{ }^{-/}$mice. This suggested that the constitutive Th2 bias required both IgE and IL-4 but not mast cells. This led us to postulate a possible role for basophils, given that they respond to an $\mathrm{IgE} / \mathrm{Ag}$ stimulus and produce large amounts of IL-4 [2, 31]. This assumption was also in light of the previously mentioned observation that $\mathrm{Lyn}^{-/-}$mice had a peripheral basophilia, with increased number of basophils present in the blood, spleen and peritoneum [22]. Ex vivo FceRI stimulation of $\mathrm{Lyn}^{-/-}$and WT blood basophils demonstrated a marked increase in IL-4 production on a per cell basis by $L y n^{-/-}$basophils [22]. To test $L y n^{-/-}$basophil function in vivo, we took advantage of the previous observation that the basophil is a key initial responder upon papain immunization [34]. Strikingly, in $\mathrm{Lyn}^{-/}$mice immunization with papain led to a rapid increase in specific IgE production, which occurred within 7 days of the first immunization and differed from WT mice where specific $\operatorname{IgE}$ is normally observed after a second antigen challenge [22]. Interestingly, the control immunization with human serum albumin (HSA) also led to significant production of HSAspecific IgE whereas this antigen does not induce an effective IgE response in WT mice [34]. This demonstrated that the $\mathrm{Lyn}^{-/}$mice mounted an early and inappropriate Th2 response. To determine if this Th2 bias altered Th1 responses in these mice, we assessed their ability to respond to a Th1 challenge. WT and $\mathrm{Lyn}^{-/}$mice were infected with Toxoplasma gondii, an intracellular pathogen that initiates a host Th1 response and whose clearance is strictly dependent on IFN $\gamma$ [66]. A week after infection, the levels of IFN $\gamma$ in the sera of $\mathrm{Lyn}^{-/}$mice were about one-half of those in WT mice, and by 1 month of infection $L_{y n}{ }^{-/-}$mice had a markedly increased accumulation of cysts in the brain relative to WT mice [22].

While the aforementioned findings provided some evidence for a role of basophils in the Th2 bias of $\mathrm{Lyn}^{-/-}$mice, it was all circumstantial. In order to confirm the role of basophils in the constitutive Th2 skewing of these mice, we adopted two distinct approaches. First, basophils were stimulated in vivo via FceRI in $W^{S h} / W^{S h} L y n^{-/-}$and $W^{S h} / W^{S h}$ mice. We reasoned that since these mice are mast cell deficient and have high IgE levels, the basophils would be saturated by $\mathrm{IgE}$ and injection of rat anti-mouse $\operatorname{IgE}$ would selectively target the basophil. Also anaphylaxis would not be an issue in mast cell-deficient mice, since IgE-mediated passive systemic anaphylaxis is mast cell dependent [67]. This challenge resulted in a strong Th2 skewing of $W^{S h} / W^{S h}$ mice similar to the constitutive Th2 skewing of $\mathrm{Lyn}^{-/}$mice. For $W^{S h} / W^{S h} \mathrm{Lyn}^{-/-}$mice, the Th2 skewing was even more exacerbated than in unstimulated mice, with up to $35 \%$ of $\mathrm{CD}^{+}$ splenocytes producing IL-4 (in the absence of re-stimulation with PMA-ionomycin) [22]. These findings demonstrated that FceRI-dependent stimulation of basophils in vivo resulted in a potent induction of naïve $\mathrm{CD} 4^{+} \mathrm{T}$ cell differentiation to Th2 cells. Importantly, we did not observe a Th1 response after the in vivo stimulation of basophils, showing that the activated basophil induced a selective Th2 differentiation [22]. To further confirm these findings we adopted the opposite approach of depleting basophils in WT and $\mathrm{Lyn}^{-/-}$mice. Depletion of basophils in vivo from $\mathrm{Lyn}^{-/-}$mice using the basophil depleting antibody MAR-1, as mentioned above, led to the complete reversal of the Th2 skewing seen in these mice. Mice treated in this manner showed normal $\mathrm{CD}^{+}$naïve $\mathrm{T}$ cells and no presence of $\mathrm{Th} 2$ cells in the spleen. The constitutive Th2 skewing was also reversed in $W^{S h} / W^{S h} L y n^{-/-}$mice by depleting basophils from these mice. Thus, the results described in this section demonstrate that the peripheral basophilia seen in $L y n^{-/-}$mice leads to the constitutive Th2 cell differentiation seen in these mice and the presence of IL-4 and IgE is required for this effect [22].

\section{Linking the Th2 Environment to Autoimmunity}

It is well known that SLE is a multifactorial autoimmune disease affecting multiple organs. This autoimmune disease affects mainly women in childbearing age (15-40 years old) and can be lethal when kidney involvement (lupus nephritis) is severe and non-responsive to treatment [68]. The etiology of SLE is unknown but it is clear that both environmental and genetic factors play a role in the development of the disease [68]. The American Rheumatology Association (ARA) established 11 distinct criteria to guide physicians in the diagnosis of SLE, based on the clinical manifestations in patients [69]. Presence of at least 4 of the 11 criteria is needed for a definitive diagnosis. Among the criteria is the produc- 
tion of autoantibodies to double stranded DNA (dsDNA) and anti-nuclear antigens (ANA) [69], a feature found in other autoimmune diseases. Like in the mouse, these autoantibodies cause the formation of circulating immune complexes (CIC), deposits in the kidney and the chronic inflammation that leads to tissue damage and renal failure. Treatment of SLE currently relies on non-specific immunosuppression and steroids, making new therapeutic approaches of particular interest.

Recently, a polymorphism in an untranslated region of the gene encoding Lyn kinase has been associated with some population of SLE patients and was specifically shown to be correlated with increased autoantibody production [70]. Expression of Lyn kinase was also shown to be lower in the peripheral B cell compartment of some SLE patients relative to normals [71]. Thus, Lyn kinase seems to play a role in SLE and it is not surprising that in late life (after 20 weeks of age) $\mathrm{Lyn}^{-/}$mice develop an autoimmune phenotype that shows many of the features of human SLE. $\mathrm{Lyn}^{-/-}$mice develop high levels of autoantibodies specific for double stranded DNA (dsDNA) and nuclear antigens (ANA) with resulting high amounts of circulating immune complexes (CIC) in their serum [18, 19]. Like in human SLE (see below), CICs of IgG- IgA- and IgM- subclasses are deposited in the glomeruli of the kidney of $\mathrm{Lyn}^{-/}$mice leading to the development of a chronic lupus nephritis-like disease, increasing tissue injury, loss of kidney function, and death as mice age $[18,19]$. This suggested that the $\mathrm{Lyn}^{-/-}$mice could be a suitable model for exploring the effect of a Th2 environment in the development of a lupus-like disease. In particular, given the early life Th2 skewing in these mice and the late life development of SLE in $\mathrm{Lyn}^{-/-}$mice independent of genetic background, the argument could be made that the constitutive Th2 environment was permissive to a disease thought to be primarily mediated by Th1 and Th17 cells.

\section{Development of Th2-driven Lupus Nephritis}

Aged $\mathrm{Lyn}^{-/}$mice (over 30 weeks old) showed a constitutive Th2 skewing without any apparent increase in Th1 responses as already described in younger mice [22]. At this late age, $\mathrm{Lyn}^{-/-}$mice have hallmarks of an autoimmune lupuslike disease with remarkably high levels of autoantibodies and kidney disease $[18,19,49]$. The penetrance of disease was complete with all $\mathrm{Lyn}^{-/-}$mice developing some feature of autoimmunity. To investigate if the Th2 environment contributed to the late life autoimmune phenotype of the $\mathrm{Lyn}^{-/}$ mice, we studied the SLE-like phenotype of the $\operatorname{Igh} 7^{-1} \mathrm{Lyn}^{-/}$ and $I l-4^{-/-} \mathrm{Lyn}^{-/-}$mice. These mice developed nearly all of the features of $\mathrm{Lyn}^{-/-}$mice, showing an altered peripheral B cell compartment, splenomegaly, hyper serum IgM and IgA concentrations, and importantly peripheral basophilia. The only difference observed in the $\mathrm{Igh} 7^{-/-} \mathrm{Lyn}^{-/-}$and $\mathrm{Il}-4^{-/-} \mathrm{Lyn}{ }^{-/-}$mice relative to $\mathrm{Lyn}^{-/}$mice was the absence of a constitutively $\mathrm{Th} 2$ skewed $\mathrm{CD}^{+} \mathrm{T}$ cell population [22]. Scoring of the glomerulonephritis (based on cellularity, mesangial proliferation, crescent formation and necrosis) in the $\operatorname{Igh} 7^{-/-} \mathrm{Lyn}{ }^{-/-}$ and $\mathrm{Il}-4^{-/-} \mathrm{Lyn}^{-/-}$mice relative to $\mathrm{Lyn}{ }^{-/-}$mice revealed that the former mice showed no obvious kidney disease. Evaluation of IgM-, IgA- and IgG-containing immune complexes in their glomeruli showed a marked reduction in their deposition in the kidney and this was associated with relatively little proteinuria. Thus, it was evident that the development of the lupus-like disease in $\mathrm{Lyn}^{-/-}$mice was dependent on the presence of IL-4 and IgE; whose absence reversed the Th2 skewing seen in these mice. Strikingly, the titers of IgG antidsDNA and ANA as well as the amounts of CIC were also decreased in $I g h 7^{-/-} \mathrm{Lyn}^{-/-}$and $I l-4^{-/-} \mathrm{Lyn}^{-/-}$mice, showing that autoantibody production in $L y n^{-/}$mice was, at least in part, dependent on the Th2 environment.

These findings suggested the involvement of basophils in autoantibody production, since we previously showed their role in promoting the Th2 skewing of $\mathrm{Lyn}^{-/-}$mice [22]. To confirm the involvement of basophils in the production of autoantibodies, we again used the antibody-mediated depletion of basophils to test their role in the production of autoantibodies. Since the depletion of basophils by this approach is transient (6-8 days), and the antibody half-life is normally 2 weeks, the finding that dsDNA and ANA IgG titers were markedly reduced (in some cases by greater than $50 \%$ ) in 40 week old $\mathrm{Lyn}^{-/-}$mice was unexpected. Even more striking was the finding that in younger (20 week old) $W^{S h} / W^{S h} \mathrm{Lyn}^{-/-}$mice, basophil depletion resulted in an almost complete loss of autoantibody production to levels seen in the control $W^{S h} / W^{S h}$ mice. One might argue that the observed decrease of autoantibodies might simply reflect the rapid clearance of immune complexes which have a much shorter serum half-life that an uncomplexed antibody [72]. On the other hand, with the continuous production of autoantibodies in autoimmune diseases, one might argue that the marked reduction in autoantibody titers could not have been solely due to the clearance of immune complexes. Analysis of plasma cell numbers in the spleen revealed that the depletion of basophils led to a large decrease (over 60\%) of plasma cells in the spleen 6 days post basophil depletion, whereas the latter had no effect on plasma cell number in WT mice. Thus, the rapid decrease in plasma cell numbers, upon basophil depletion, is likely to rapidly affect autoantibody titers. This might suggest a role of the basophil-derived IL-6, which is know to induce B cell maturation, proliferation and survival [44], in the autoantibody production occurring in $\mathrm{Lyn}^{-/-}$mice. In fact, we found that after FceRI stimulation Lyn $^{-/}$basophils were able to produce large amounts of IL-6 relative to basophils from WT mice [22]. It should be noted, that no depletion of bone marrow plasma cells was observed when basophils were depleted. This argues against a direct effect of the basophil-depleting antibody on the plasma cells, but also shows the limitation of short term depletion on long term autoantibody production. Collectively, the findings show the contribution of the basophil, IgE, and IL-4 to the maintenance of autoantibody production in $\mathrm{Lyn}^{-/-}$mice and demonstrate that the Th2 environment can be contributory in autoimmunity.

\section{Activation and Homing of Basophils in $\mathrm{Lyn}^{-/-}$Mice}

From the above results, it was clear that basophils, IgE and IL-4 were contributory factors in the autoantibody production and the lupus-like disease seen in $\mathrm{Lyn}^{-/}$mice. However, an important question still remained unanswered: How are the hyperresponsive basophils in $\mathrm{Lyn}^{-/-}$mice constitutively activated? Because disease development in $\mathrm{Lyn}^{-/-}$mice required IgE and IL-4, we postulated that the constitutive activation of basophils might result from the basophil- 
independent production of autoreactive antibodies that could cause basophil activation. Interestingly, analysis of autoantibody isotypes revealed high levels of anti-dsDNA and ANA of the IgE class in the sera from $\mathrm{Lyn}^{-/}$and $W^{S h} / W^{S h} \mathrm{Lyn}^{-/-}$ mice. This autoreactive $\mathrm{IgE}$ was absent or dramatically reduced in the sera of $I g h 7^{-/} L y n^{-/-}$and $I l-4^{-/-} \mathrm{Lyn}^{-/-}$mice, respectively. Moreover, IgE-containing CICs were present in the circulation of $\mathrm{Lyn}^{-/}$mice. Given that basophils express FceRI, it seemed reasonable to think that these IgE-CICs could be the key factor in promoting basophil activation. Nonetheless, basophils also express Fcy receptors and IgGCICs were also present in high amounts in the sera of $\mathrm{Lyn}^{-/}$ and $W^{S h} / W^{S h} \mathrm{Lyn}^{-/-}$mice. Thus, we explored the ability of IgE or IgG-containing immune complexes (ICs) to induce basophil activation. IgE-ICs were highly effective in activating basophils to produce IL-4 and IL-6 (but not IFN- $\gamma$, IFN- $\alpha$, IFN- $\beta$, TGF- $\beta$ or IL-12p40), Lyn-deficiency caused secretion of significantly higher amounts of these cytokines relative to WT basophils. In contrast, IgG-containing ICs failed to induce detectable activation of basophils and no cytokine production was observed. These findings argue that the constitutive activation of basophils in $\mathrm{Lyn}^{-\gamma}$ mice is most likely due to the presence of circulating autoreactive IgE-containing ICs, which are likely to be generated due to B cell hyperresponsiveness to normal levels of IL-4 and CD40 stimulation.

While there are several cell surface activation markers described for human basophils, such as CD203c, CD63 or CCR3 expression [73], activation markers for mouse basophils are not as well defined. Nonetheless, it was important to explore the activation status of basophils in $\mathrm{Lyn}^{--}$mice. CD62L (L-selectin) is a cell surface marker that is expressed on immune cells for their homing to peripheral lymphoid organs and tissues [74] and has been shown to be expressed on human basophils [75]. Measurement of CD62L expression on blood basophils from $\mathrm{Lyn}^{-/}$mice showed a marked upregulation of its expression relative to basophils from WT mice. Because expression of CD62L causes homing to lymphoid tissues, we asked if basophils from $\mathrm{Lyn}^{-/}$mice could be recruited to lymphoid organs, where they could presumably interact with $\mathrm{T}$ and $\mathrm{B}$ cells. This possibility might explain how $\mathrm{Lyn}^{-/-}$basophils are able to induce Th2 skewing of naïve $\mathrm{CD}^{+} \mathrm{T}$ cells as well as participate in autoantibody production and promote increased numbers of plasma cells. In aged WT control mice, basophils could not be found in lymph nodes. In contrast, in aged $\mathrm{Lyn}^{-/-}$and $W^{S h} / W^{S h} \mathrm{Lyn}^{-/-}$ mice, basophils were recruited and accumulated in lymph nodes (axial and cervical). As expected, given the diminished ability to activate basophils in aged $\operatorname{Igh} 7^{-/-} \mathrm{Lyn}^{-/-}$and Il- $4^{-/} \mathrm{Lyn}^{-/-}$mice, the lymph nodes of these mice had only a small percentage $(\sim 10 \%)$ of the basophils seen in $\mathrm{Lyn}^{-/-}$and $W^{S h} / W^{S h} L^{\prime-\gamma}{ }^{--}$mice. These findings show that basophils from $\mathrm{Lyn}^{-/-}$and $W^{S h} / W^{S h} \mathrm{Lyn}^{-/-}$mice migrate to lymphoid tissues where they can influence both $\mathrm{T}$ and $\mathrm{B}$ cells. While the production of cytokines, like IL-4 and IL-6, are important towards influencing $\mathrm{T}$ and $\mathrm{B}$ cells, we also found that basophils from $L y n^{-/-}$and $W^{S h} / W^{S h} L y n^{-/-}$mice express high levels of MHC class II molecules, and high levels of membrane bound BAFF (a B cell survival and activation factor), which has been shown to be associated with SLE [76]. Expression of membrane BAFF did not appear to be dependent on the $\mathrm{BAFF}$ receptor, which was detected at very low levels and was not upregulated. Thus, the activation of basophils by circulating IgE-ICs in $L y n^{-/-}$and $W^{S h} / W^{S h} L_{y n}{ }^{-/-}$mice results in the homing of these cells to secondary lymphoid tissues and also causes the upregulation of key surface regulatory molecules that can directly promote $\mathrm{T}$ cell differentiation and $\mathrm{B}$ cell differentiation and survival. This process is markedly dampened in the absence of IgE or IL-4, as evidenced in $\operatorname{Igh} 7^{-/} \mathrm{Lyn}^{-/-}$and $\mathrm{Il}-4^{-/-} \mathrm{Lyn}^{-/-}$mice.

The data generated from our mouse studies has allowed us to propose a model (Fig. 1) of how basophils may act to amplify autoreactive responses. We show that in the spontaneous lupus model of $\mathrm{Lyn}^{-/-}$mice, autoantibodies (dsDNA and ANA) are produced by autoreactive B cells, likely through help provided by autoreactive T cells. How this is initiated is not known, however, the production of autoreactive IgE, likely in response to IL-4 and/or CD40 stimulation, causes basophil activation. IL-4 production by basophils amplifies the production of autoantibodies by promoting increased Th2 cell differentiation and increasing B cell activation and survival, including the survival of plasma cells in the spleen. This is mediated through increased CD62L expression on basophils, which causes their migration and accumulation in peripheral lymphoid tissues, and the cell surface expression of mBAFF and MHC-II expression on basophils in these tissues. Thus, the data from the $\mathrm{Lyn}^{-/-}$mouse model argues that the basophil is an important contributor in the development and maintenance of lupus-like disease in these mice. Whether this model (Fig. 1) is applicable to the disease seen in other spontaneous models of SLE (like MRL/lpr, NZBW, or BSXB mice) remains to be determined. While most of these models have been shown to have a Th1 and/or Th17 component, it is notable that these mice have been described to have increased levels of circulating IgE [77], suggesting that a Th2 component may contribute to the pathology.

\section{Basophil Dependent Kidney Inflammation}

Damage to kidney function in severe lupus nephritis is thought to be irreversible due to the inflammation that causes severe injury and structural alterations to the kidney [78]. Thus, "long term" basophil depletion (over three weeks, with repeated injections of basophil depleting antibody) in 40 week old $\mathrm{Lyn}^{-/-}$mice did not lead to improvement of renal function (unpublished data). Nonetheless, we could explore whether basophil depletion might alter the inflammatory environment in the diseased kidney of $\mathrm{Lyn}^{-/-}$mice. Antibodymediated depletion of basophils was found to cause a marked decrease in pro-inflammatory cytokines detected in the kidney of aged $\mathrm{Lyn}^{-/}$mice. Within six days after depletion, a decrease of IL- $1 \beta$, IL-4, IL-6, and IFN- $\gamma$ was found with as much as a $70 \%$ reduction seen for some cytokines. This demonstrated that basophils (and likely their ability to control autoantibody production) are contributors to the proinflammatory environment found in the lupus kidney. Additionally, we found that in the $\operatorname{Igh} 7^{-/} \mathrm{Lyn}^{-/-}$mice (which did not have renal failure and showed reduced CIC deposition in the kidney) the pro-inflammatory cytokine environment in the kidney was greatly reduced. Collectively, the findings demonstrate that basophils contribute to the kidney inflammation that occurs in lupus nephritis. Thus, it is of considerable interest to explore if basophil depletion or inactivation, 


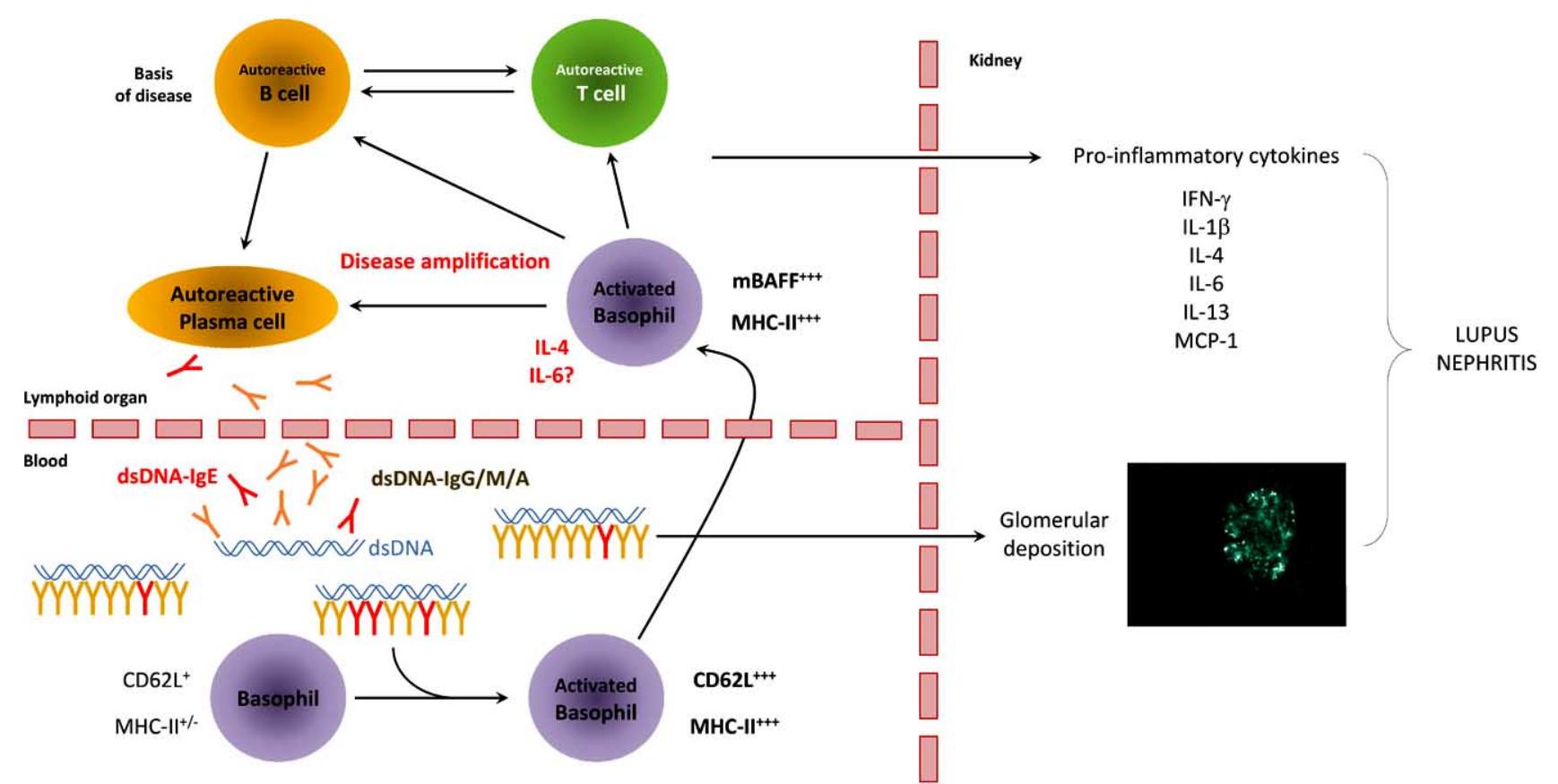

Fig. (1). Basophils function in the amplification of autoantibody production in SLE.

The accepted basis of disease in SLE is an escape of autoreactive B and T cells from negative selection (for reasons still not completely understood). These autoreactive lymphocytes lead to the production of autoreactive antibodies "aggregating" with complement factors and autoantigens in the form of circulating immune complexes (CICs). Autoreactive IgGs, IgMs and IgAs being the majority of them, autoreactive IgE, in some circumstances, could accumulate in the CICs. These IgE containing CICs then activate circulating blood basophils leading to their recruitment to lymphoid organs via the CD62L. Once recruited, basophils, via MHC-II and membrane bound BAFF (mBAFF) interact directly with autoreactive lymphocytes. Via their ability to secrete large amounts of IL-4 (and IL-6), basophils then amplify the autoantibody production, leading to the disease amplification.

at an early stage of disease, could delay or rescue the early development of lupus nephritis.

\section{BASOPHILS AND THE Th2 ENVIRONMENT IN HU- MAN SLE}

While mouse models are useful to explore mechanistic and/or physiological aspects that one cannot explore in human disease, the translation of the findings in mouse models to human disease must be explored [63]. Thus, we set out to determine if SLE patients had circulating autoreactive antibodies (anti-dsDNA IgE or anti-IgE IgGs) that might be able to stimulate basophils. As previously mentioned, these autoreactive antibodies were already described to be present in SLE and urticaria's [23, 50]. However, it was unclear as to whether all SLE patients generated such antibodies. Analysis of plasma samples from a cohort of 43 patients demonstrated the presence of IgE with specificity to dsDNA in all SLE patients screened. When we categorized the patients in 3 distinct groups based on disease activity or SLEDAI score (SLE disease activity index, inactive SLEDAI=0-1, mild SLEDAI $=2-4$ and active SLEDAI $>4$ ), the levels of antidsDNA IgE were strongly associated with increasing disease activity. IgG anti-IgE antibodies were found only in patients with mild or active disease and their levels were also associated with increased disease activity. When patient antidsDNA IgE levels were analyzed on the basis of the absence or presence of active nephritis, a striking 4 fold increase in the amount of anti-dsDNA $\operatorname{IgE}$ was seen in patients with active nephritis compared to those without active kidney disease. Thus, these findings suggest the direct involvement of autoreactive IgE in human lupus nephritis. Since previous studies [23, 55] had demonstrated that SLE patients had increased circulating $\operatorname{IgE}$, we explored if total $\operatorname{IgE}$ was increased in our cohort of patients. While not all patients had increased levels of IgE (whereas all had anti-dsDNA IgE), in patients where total IgE was increased, an association with disease activity and active lupus nephritis was noted. Collectively, the data from human SLE patients demonstrates that beyond the role of Th1 or Th17 cells in this disease, SLE patients also manifest a Th2 component that contributes to increased disease activity and active nephritis.

These findings led us to explore the activation status of blood basophils in SLE patients and healthy (normal) controls. This was based on the assumption that the presence of anti-dsDNA IgE or anti-IgE IgG might lead to basophil activation in these patients. As in the $\mathrm{Lyn}^{-/}$mouse model, this could cause permanent activation of circulating basophils, via stimulation of FceRI, leading to amplification of autoantibody production and lupus nephritis. In human basophils, CD203c is known to be expressed upon activation of these cells [79]. Our analysis of CD203c expression on basophils from SLE patients and healthy controls revealed a significant upregulation of this cell surface molecule on blood basophils from SLE patients relative to controls. In addition, as observed in the $\mathrm{Lyn}^{-/}$mouse model, blood basophils from SLE patients also had increased levels of CD62L on their surface, and the increased CD62L expression correlated with disease activity. This suggested the possibility that, like in the mouse 
model, basophils from SLE patients might be found in the peripheral lymphoid organs such as spleen and lymph nodes. Analysis of biopsies from the spleen and lymph nodes of SLE patients and healthy controls revealed a striking difference in basophil homing, with basophils being present at both sites for SLE patients whereas none were detected in the control tissue. In the patient lymph node, basophils accumulated in the periphery or the B cell rich zone of germinal centers, demonstrating that they localize to a site where they could influence both $\mathrm{T}$ and $\mathrm{B}$ cells. In contrast, we did not find the presence of basophils in kidney biopsies from SLE patients with active nephritis or healthy controls. This could not be analyzed in the $\mathrm{Lyn}^{-/-}$mouse model due to the lack of a suitable reagent for basophil detection in mice. This suggests that the role of the basophil may be immunomodulatory in this disease rather than as a pro-inflammatory effector. Consistent with the homing of basophils to secondary lymphoid tissues, we observed a significant decrease in the number of circulating basophils in SLE patients, and this decrease was associated with increased disease activity. A decrease in the numbers of circulating basophils was not observed in $\mathrm{Lyn}^{-/}$mice, however, the mice had a peripheral basophilia (driven by the absence of Lyn [22]) and this was not seen in patients. Thus, in the $L y n^{-/-}$mouse, the dysregulation of basophil proliferation likely masks the depletion of basophils from the circulation when basophils homed to secondary lymphoid tissues. Unlike the mice, Lyn expression in the basophils of SLE patients was normal when compared to healthy controls.

An alternate explanation for the loss of basophils from the blood of SLE patients could be the immunosuppressive treatment that these patients undergo. While we found that corticosteroids, like prednisone, showed no association with the loss of blood basophils, combination immunosuppressive treatments were associated with decreased blood basophil numbers. However, the immunosuppressive treatments had no apparent effect on the increased basophil activation in SLE patients. This suggests that immunosuppressive treatments may work, in part, through their suppression of blood basophils. This indicates that one might consider the depletion of basophils from the blood of SLE patients as a potential therapeutic strategy. Since the key may well be to diminish basophil activation, another approach might be to deplete $\operatorname{IgE}$ (and thus dsDNA-IgE) in SLE patients. This latter approach could be tested rapidly, since the IgE-depleting antibody omalizumab (Xolair ${ }^{\circledR}$, Genentech), is currently in use for treatment of asthma and allergic rhinitis in some patients and is seemingly well tolerated $[80,81]$. Thus, the finding of a role for basophils in SLE [82] may open new avenues with therapeutic potential in the treatment of this disease.

\section{CONCLUDING REMARKS}

As described herein, we are at the beginning stages of unraveling the immunomodulatory and effector functions of basophils in health and disease. However, it can be said that beyond their role in allergy and in host defense to parasites, the basophil can promote Th2 cell differentiation and function to amplify the humoral immune response. Its role in autoimmune diseases has not previously been recognized, but the aforementioned findings [82] make a strong argument for a role in lupus nephritis. This role is not in initiation but instead amplification of disease. Key requirements are the autoreactive IgE-dependent activation of basophils, the production of IL-4 by these cells, and the promotion of Th2 cell differentiation. This does not preclude the demonstrated role for Th1, Th17, and the loss of regulatory $\mathrm{T}$ cell function, in the development of autoimmunity. Instead, it argues that the strong humoral component seen in autoimmune diseases, like SLE, requires the basophil in order to reach the levels of autoantibody production that result in kidney disease and sometimes kidney failure. Our findings in $\mathrm{Lyn}^{-/-}$mice, as well as in SLE patients, point to the Th2 environment as contributory in SLE.

The recent advances in our understanding of the role of basophils in health and disease demonstrate that a small population of basophil granulocytes can have an important immunomodulatory role in the immune system. However, one must be cautious with the over enthusiastic view of the basophil as necessary for Th2 cell differentiation. To date, experiments dealing with this issue are limited and the key test of challenging mice with a Th2 antigen in the complete absence of basophils remains to be done. Nonetheless, the findings show that in some settings the basophil is a potent inducer of Th2 cell differentiation and through this ability it links the "allergic" environment to the development of an autoimmune disease, like SLE. It will be of interest to explore if these findings will translate to therapeutic benefit.

\section{ACKNOWLEDGEMENTS}

The research of the authors cited herein was supported by the intramural program of the National Institute of Arthritis and Musculoskeletal and Skin Diseases (NIAMS) of the National Institutes of Health (NIH). We also acknowledge the support of the Laboratory Animal Care and Use Section, the Light Imaging Section, and the Flow Cytometry Section, Office of Science and Technology, NIAMS.

\section{REFERENCES}

[1] Maizels RM, Pearce EJ, Artis D, Yazdanbakhsh M, Wynn TA. Regulation of pathogenesis and immunity in helminth infections. J Exp Med 2009; 206(10): 2059-66.

[2] Min B, Paul WE. Basophils and type 2 immunity. Curr Opin Hematol 2008; 15(1): 59-63.

[3] Schroeder JT, Kagey-Sobotka A, Lichtenstein LM. The role of the basophil in allergic inflammation. Allergy 1995; 50(6): 463-72.

[4] Mukai K, Matsuoka K, Taya C, et al. Basophils play a critical role in the development of IgE-mediated chronic allergic inflammation independently of $\mathrm{T}$ cells and mast cells. Immunity 2005; 23(2): 191-202.

[5] Tsujimura Y, Obata K, Mukai K, et al. Basophils play a pivotal role in immunoglobulin-G-mediated but not immunoglobulin-Emediated systemic anaphylaxis. Immunity 2008; 28(4): 581-9.

[6] Karasuyama H, Mukai K, Tsujimura Y, Obata K. Newly discovered roles for basophils: a neglected minority gains new respect. Nat Rev Immunol 2009; 9(1): 9-13.

[7] Min B. Basophils: what they 'can do' versus what they 'actually do'. Nat Immunol 2008; 9(12): 1333-9.

[8] Rivera J, Gilfillan AM. Molecular regulation of mast cell activation. J Allergy Clin Immunol 2006; 117(6): 1214-25; quiz 26.

[9] Gilfillan AM, Rivera J. The tyrosine kinase network regulating mast cell activation. Immunol Rev 2009; 228(1): 149-69.

[10] Odom S, Gomez G, Kovarova M, et al. Negative regulation of immunoglobulin E-dependent allergic responses by Lyn kinase. J Exp Med 2004; 199(11): 1491-502.

[11] Kovarova M, Wassif CA, Odom S, Liao K, Porter FD, Rivera J Cholesterol deficiency in a mouse model of Smith-Lemli-Opitz syndrome reveals increased mast cell responsiveness. J Exp Med 2006; 203(5): 1161-71. 
[12] Yamashita Y, Charles N, Furumoto Y, et al. Cutting edge: genetic variation influences $\mathrm{Fc}$ epsilonRI-induced mast cell activation and allergic responses. J Immunol 2007; 179(2): 740-3.

[13] Xiao W, Nishimoto H, Hong H, et al. Positive and negative regulation of mast cell activation by Lyn via the FcepsilonRI. J Immunol 2005; 175(10): 6885-92.

[14] Hong H, Kitaura J, Xiao W, et al. The Src family kinase Hck regulates mast cell activation by suppressing an inhibitory Src family kinase Lyn. Blood 2007; 111: 2511-9.

[15] Kitaura J, Kawakami Y, Maeda-Yamamoto M, Horejsi V, Kawakami T. Dysregulation of Src family kinases in mast cells from epilepsy-resistant ASK versus epilepsy-prone EL mice. J Immunol 2007; 178(1): 455-62.

[16] Hernandez-Hansen V, Mackay GA, Lowell CA, Wilson BS, Oliver JM. The Src kinase Lyn is a negative regulator of mast cell proliferation. J Leukoc Biol 2004; 75(1): 143-51.

[17] Hernandez-Hansen V, Smith AJ, Surviladze Z, et al. Dysregulated FcepsilonRI signaling and altered Fyn and SHIP activities in Lyndeficient mast cells. J Immunol 2004; 173(1): 100-12.

[18] Hibbs ML, Tarlinton DM, Armes J, et al. Multiple defects in the immune system of Lyn-deficient mice, culminating in autoimmune disease. Cell 1995; 83(2): 301-11.

[19] Nishizumi H, Taniuchi I, Yamanashi Y, et al. Impaired proliferation of peripheral $\mathrm{B}$ cells and indication of autoimmune disease in lyn-deficient mice. Immunity 1995; 3(5): 549-60.

[20] Beavitt SJ, Harder KW, Kemp JM, et al. Lyn-deficient mice develop severe, persistent asthma: Lyn is a critical negative regulator of Th2 immunity. J Immunol 2005; 175(3): 1867-75.

[21] Yamanashi Y, Mori S, Yoshida M, et al. Selective expression of a protein-tyrosine kinase, p56lyn, in hematopoietic cells and association with production of human T-cell lymphotropic virus type I. Proc Natl Acad Sci U S A 1989; 86(17): 6538-42.

[22] Charles N, Watford WT, Ramos HL, et al. Lyn kinase controls basophil GATA-3 transcription factor expression and induction of Th2 cell differentiation. Immunity 2009; 30(4): 533-43.

[23] Atta AM, Sousa CP, Carvalho EM, Sousa-Atta ML. Immunoglobulin $\mathrm{E}$ and systemic lupus erythematosus. Braz $\mathrm{J}$ Med Biol Res 2004; 37(10): 1497-501.

[24] Balomenos D, Rumold R, Theofilopoulos AN. Interferon-gamma is required for lupus-like disease and lymphoaccumulation in MRLlpr mice. J Clin Invest 1998; 101(2): 364-71.

[25] Masutani K, Akahoshi M, Tsuruya K, et al. Predominance of Th1 immune response in diffuse proliferative lupus nephritis. Arthritis Rheum 2001; 44(9): 2097-106.

[26] Zeng D, Liu Y, Sidobre S, Kronenberg M, Strober S. Activation of natural killer $\mathrm{T}$ cells in NZB/W mice induces Th1-type immune responses exacerbating lupus. J Clin Invest 2003; 112(8): 1211-22.

[27] Peng SL, Moslehi J, Craft J. Roles of interferon-gamma and interleukin-4 in murine lupus. J Clin Invest 1997; 99(8): 1936-46.

[28] Peng SL, Szabo SJ, Glimcher LH. T-bet regulates IgG class switching and pathogenic autoantibody production. Proc Natl Acad Sci U S A 2002; 99(8): 5545-50.

[29] Valencia X, Yarboro C, Illei G, Lipsky PE. Deficient $\mathrm{CD} 4+\mathrm{CD} 25$ high $\mathrm{T}$ regulatory cell function in patients with active systemic lupus erythematosus. J Immunol 2007; 178(4): 2579-88.

[30] Pernis AB. Th17 cells in rheumatoid arthritis and systemic lupus erythematosus. J Intern Med 2009; 265(6): 644-52.

[31] Yoshimoto T, Yasuda K, Tanaka H, et al. Basophils contribute to $\mathrm{T}(\mathrm{H}) 2-\mathrm{IgE}$ responses in vivo via IL-4 production and presentation of peptide-MHC class II complexes to CD4+ T cells. Nat Immunol 2009; 10(7): 706-12.

[32] Perrigoue JG, Saenz SA, Siracusa MC, et al. MHC class IIdependent basophil-CD4+ $\mathrm{T}$ cell interactions promote $\mathrm{T}(\mathrm{H}) 2$ cytokine-dependent immunity. Nat Immunol 2009; 10(7): 697-705.

[33] Sokol CL, Chu NQ, Yu S, Nish SA, Laufer TM, Medzhitov R. Basophils function as antigen-presenting cells for an allergeninduced T helper type 2 response. Nat Immunol 2009; 10(7): 71320.

[34] Sokol CL, Barton GM, Farr AG, Medzhitov R. A mechanism for the initiation of allergen-induced $\mathrm{T}$ helper type 2 responses. Nat Immunol 2008; 9(3): 310-8.

[35] Denzel A, Maus UA, Rodriguez Gomez M, et al. Basophils enhance immunological memory responses. Nat Immunol 2008; 9(7): 733-42.

[36] Chen Z, O'Shea JJ. Th17 cells: a new fate for differentiating helper T cells. Immunol Res 2008; 41(2): 87-102.
[37] Weiss DL, Brown MA. Regulation of IL-4 production in mast cells: a paradigm for cell-type-specific gene expression. Immunol Rev 2001; 179: 35-47.

[38] Zhu J, Paul WE. CD4 T cells: fates, functions, and faults. Blood 2008; 112(5): 1557-69.

[39] Kadowaki N. Dendritic cells: a conductor of T cell differentiation. Allergol Int 2007; 56(3): 193-9.

[40] Suzukawa M, Iikura M, Koketsu R, et al. An IL-1 cytokine member, IL-33, induces human basophil activation via its ST2 receptor. J Immunol 2008; 181(9): 5981-9.

[41] Obata K, Mukai K, Tsujimura Y, et al. Basophils are essential initiators of a novel type of chronic allergic inflammation. Blood 2007; 110(3): 913-20.

[42] Armitage RJ, Macduff BM, Spriggs MK, Fanslow WC. Human B cell proliferation and Ig secretion induced by recombinant CD40 ligand are modulated by soluble cytokines. J Immunol 1993; 150(9): 3671-80.

[43] Acosta-Rodriguez EV, Merino MC, Montes CL, Motran CC, Gruppi A. Cytokines and chemokines shaping the B-cell compartment. Cytokine Growth Factor Rev 2007; 18(1-2): 73-83.

[44] Matsuda T, Yamasaki K, Taga T, Hirano T, Kishimoto T. Current concepts of B cell modulation. Int Rev Immunol 1989; 5(2): 97109.

[45] Schroeder JT, MacGlashan DW, Jr., Lichtenstein LM. Human basophils: mediator release and cytokine production. Adv Immunol 2001; 77: 93-122.

[46] Ohnmacht C, Voehringer D. Basophil effector function and homeostasis during helminth infection. Blood 2009; 113(12): 281625.

[47] Hida S, Tadachi M, Saito T, Taki S. Negative control of basophil expansion by IRF-2 critical for the regulation of Th1/Th2 balance. Blood 2005; 106(6): 2011-7.

[48] Taki S. Type I interferons and autoimmunity: lessons from the clinic and from IRF-2-deficient mice. Cytokine Growth Factor Rev 2002; 13(4-5): 379-91.

[49] Yu CC, Yen TS, Lowell CA, DeFranco AL. Lupus-like kidney disease in mice deficient in the src family tyrosine kinases Lyn and Fyn. Curr Biol 2001; 11: 34-8.

[50] Grattan CE, Francis DM, Hide M, Greaves MW. Detection of circulating histamine releasing autoantibodies with functional properties of anti-IgE in chronic urticaria. Clin Exp Allergy 1991; 21(6): 695-704.

[51] Grattan CE. Basophils in chronic urticaria. J Investig Dermatol Symp Proc 2001; 6(2): 139-40.

[52] Bischoff SC, Zwahlen R, Stucki M, et al. Basophil histamine release and leukotriene production in response to anti-IgE and antiIgE receptor antibodies. Comparison of normal subjects and patients with urticaria, atopic dermatitis or bronchial asthma. Int Arch Allergy Immunol 1996; 110(3): 261-71.

[53] Nigrovic PA, Lee DM. Synovial mast cells: role in acute and chronic arthritis. Immunol Rev 2007; 217: 19-37.

[54] Damsker JM, Hansen AM, Caspi RR. Th1 and Th17 cells: adversaries and collaborators. Ann N Y Acad Sci 2010; 1183: 211-210.

[55] Sekigawa I, Yoshiike T, Iida N, Hashimoto H, Ogawa H. Allergic diseases in systemic lupus erythematosus: prevalence and immunological considerations. Clin Exp Rheumatol 2003; 21(1): 117-21.

[56] Shimizu S, Sugiyama N, Masutani K, et al. Membranous glomerulonephritis development with Th2-type immune deviations in MRL/lpr mice deficient for IL-27 receptor (WSX-1). J Immunol 2005; 175(11): 7185-92.

[57] Veldhoen M. The role of $\mathrm{T}$ helper subsets in autoimmunity and allergy. Curr Opin Immunol 2009; 21(6): 606-11.

[58] Black P. Why is the prevalence of allergy and autoimmunity increasing? Trends Immunol 2001; 22(7): 354-5.

[59] Martin F, Chan AC. Pathogenic roles of B cells in human autoimmunity; insights from the clinic. Immunity 2004; 20(5): 517 27.

[60] Valenta R, Seiberler S, Natter S, et al. Autoallergy: a pathogenetic factor in atopic dermatitis? J Allergy Clin Immunol 2000; 105(3): 432-7.

[61] Chen $\mathrm{K}, \mathrm{Xu} \mathrm{W}$, Wilson $\mathrm{M}$, et al. Immunoglobulin $\mathrm{D}$ enhances immune surveillance by activating antimicrobial, proinflammatory and B cell-stimulating programs in basophils. Nat Immunol 2009; 10(8): 889-98. 
[62] Hibbs ML, Harder KW. The duplicitous nature of the Lyn tyrosine kinase in growth factor signaling. Growth Factors 2006; 24(2): $137-49$.

[63] Rivera J, Tessarollo L. Genetic background and the dilemma of translating mouse studies to humans. Immunity 2008; 28(1): 1-4

[64] Parravicini V, Gadina M, Kovarova M, et al. Fyn kinase initiates complementary signals required for IgE-dependent mast cell degranulation. Nat Immunol 2002; 3(8): 741-8.

[65] Ren CL, Morio T, Fu SM, Geha RS. Signal transduction via CD40 involves activation of lyn kinase and phosphatidylinositol-3-kinase, and phosphorylation of phospholipase C gamma 2. J Exp Med 1994; 179(2): 673-80.

[66] Scharton-Kersten TM, Wynn TA, Denkers EY, et al. In the absence of endogenous IFN-gamma, mice develop unimpaired IL-12 responses to Toxoplasma gondii while failing to control acute infection. J Immunol 1996; 157(9): 4045-54.

[67] Olivera A, Eisner C, Kitamura Y, et al. Sphingosine kinase 1 and sphingosine-1-phosphate receptor 2 are vital to recovery from anaphylactic shock. J Clin Invest 2010; 120(5): 1429-40.

[68] Rahman A, Isenberg DA. Systemic lupus erythematosus. N Engl J Med 2008; 358(9): 929-39.

[69] Tan EM, Cohen AS, Fries JF, et al. The 1982 revised criteria for the classification of systemic lupus erythematosus. Arthritis Rheum 1982; 25(11): 1271-7.

[70] Lu R, Vidal GS, Kelly JA, et al. Genetic associations of LYN with systemic lupus erythematosus. Genes Immun 2009; 10(5): 397-403.

[71] Flores-Borja F, Kabouridis PS, Jury EC, Isenberg DA, Mageed RA. Decreased Lyn expression and translocation to lipid raft signaling domains in B lymphocytes from patients with systemic lupus erythematosus. Arthritis Rheum 2005; 52(12): 3955-65.

[72] Sigounas G, Harindranath N, Donadel G, Notkins AL. Half-life of polyreactive antibodies. J Clin Immunol 1994; 14(2): 134-40.

[73] Uguccioni M, Mackay CR, Ochensberger B, et al. High expression of the chemokine receptor CCR3 in human blood basophils. Role in activation by eotaxin, MCP-4, and other chemokines. J Clin Invest 1997; 100(5): 1137-43.

[74] Uchimura K, Rosen SD. Sulfated L-selectin ligands as a therapeutic target in chronic inflammation. Trends Immunol 2006; 27(12): 559-65.

[75] Bochner BS, Schleimer RP. Mast cells, basophils, and eosinophils: distinct but overlapping pathways for recruitment. Immunol Rev 2001; 179: 5-15.

[76] Levesque MC. Translational Mini-Review Series on B CellDirected Therapies: Recent advances in B cell-directed biological therapies for autoimmune disorders. Clin Exp Immunol 2009; 157(2): 198-208.

[77] Miyajima H, Abe K, Ushiyama C, Okumura K, Ovary Z, Hirano T. IgE allotypes in sera of mice with autoimmune diseases and in mice with graft-versus-host disease after transfusion or bone marrow transplantation. Int Arch Allergy Immunol 1996; 111(2): 152-5.

[78] Seshan SV, Jennette JC. Renal disease in systemic lupus erythematosus with emphasis on classification of lupus glomerulonephritis: advances and implications. Arch Pathol Lab Med 2009; 133(2): 233-48.

[79] Buhring HJ, Streble A, Valent P. The basophil-specific ectoenzyme E-NPP3 (CD203c) as a marker for cell activation and allergy diagnosis. Int Arch Allergy Immunol 2004; 133(4): 317-29.

[80] Kovarova M, Rivera J. A molecular understanding of mast cell activation and the promise of anti-allergic therapeutics. Curr Med Chem 2004; 11(15): 2083-91.

[81] Lin H, Boesel KM, Griffith DT, et al. Omalizumab rapidly decreases nasal allergic response and FcepsilonRI on basophils. J Allergy Clin Immunol 2004; 113(2): 297-302.

[82] Charles N, Hardwick D, Daugas E, Illei GG, Rivera J. Basophils and the $\mathrm{T}$ helper 2 environment can promote the development of lupus nephritis. Nat Med 2010; 16(6): 701-7.

(c) Charles and Rivera; Licensee Bentham Open.

This is an open access article licensed under the terms of the Creative Commons Attribution Non-Commercial License (http://creativecommons.org/licenses/by-nc/3.0/) which permits unrestricted, non-commercial use, distribution and reproduction in any medium, provided the work is properly cited. 